\title{
NEGATIVE ASSOCIATION DOES NOT IMPLY LOG-CONCAVITY OF THE RANK SEQUENCE
}

\author{
KLAS MARKSTRÖM,* Umeå University
}

\begin{abstract}
We present a minimum counterexample to the conjecture that a negatively associated random variable has an ultra-log-concave rank sequence. The rank sequence does not in fact even need to be unimodal.
\end{abstract}

Keywords: Negative association; rank sequence

2000 Mathematics Subject Classification: Primary 62H20; 60E05

Random variables with positive association are so well known that their usefulness hardly needs promotion. The properties of random variables which are negatively associated, such as Fermi-Dirac statistics and the uniform spanning tree of a graph, are on the other hand far less understood, but potentially highly useful too. Pemantle [3] started an exploration of negatively associated random variables and gave a number of results and conjectures about their structure. In this paper we aim to prove that one of these conjectures is false. After the first version of this note appeared, the author was informed that counterexamples to several of these conjectures have also been found independently by Borcea et al. [1].

Let us recall some of the relevant definitions. We let $B_{n}$ denote the Boolean lattices of subsets of $\{1, \ldots, n\}$.

Definition 1. A probability measure $\mu$ on $B_{n}$ is negatively associated if every pair of increasing functions $f: A \rightarrow \mathbb{R}^{+}$and $g:\left(B_{n} \backslash A\right) \rightarrow \mathbb{R}^{+}$satisfies

$$
C(f, g):=\int f g \mathrm{~d} \mu-\int f \mathrm{~d} \mu \int g \mathrm{~d} \mu \leq 0 .
$$

Likewise, we say that $\mu$ is strictly negatively associated if we can take the strict inequality above.

Since any increasing function can be written as a linear combination of indicator functions of upsets, we see that it suffices for (1) to hold for all such pairs of indicator functions.

Definition 2. The rank sequence of a measure $\mu$ on $B_{n}$ is the sequence

$$
a_{k}=\mu(|X|=k), \quad k=0, \ldots, n,
$$

i.e. $a_{k}$ is the probability that $X$ has size $k$.

Recall that a positive sequence is $\log$-concave if $a_{i}^{2} \geq a_{i-1} a_{i+1}$. In [3] a sequence $a_{0}, \ldots, a_{n}$ is defined to be ultra-log-concave (ULC) if the sequence $a_{k} /\left(\begin{array}{l}n \\ k\end{array}\right)$ is log-concave.

Received 16 October 2007.

* Postal address: Department of Mathematics and Mathematical Statistics, Umeå University, SE-901 87 Umeå, Sweden.

Email address: klas.markstrom@math.umu.se 
Pemantle [3] proved that an exchangeable measure on $B_{n}$ is conditionally negatively associated, meaning that all measures obtained by conditioning on the value of one variable are negatively associated if and only if its rank sequence is ULC. Furthermore, Pemantle [3] gave several conjectures on the connection between negative association and ULC rank sequences. One of these conjectures was verified in [2], where it was proved that the convolution of two ULC sequences is also ULC.

The conjecture which concerns us here is stated as follows.

Conjecture 1. ([3].) If $\mu$ is negatively associated and the set of indices for which the rank sequence is nonzero forms an interval then the rank sequence is ULC.

It is easy to check that the conjecture holds for measures defined on $B_{2}$, but we will now give examples which show that this conjecture does not hold on $B_{3}$.

Example 1. Our first example is a measure $\mu_{1}$ on $B_{3}$, which is negatively associated and has $a_{1}=a_{4}=0$ while $a_{2}$ and $a_{3}$ are nonzero. Here the nonzero elements of the rank sequence do not form an interval and so the example does not contradict Pemantle's conjecture.

Our measure is defined as

$$
\begin{gathered}
\mu_{1}(\varnothing)=\frac{6}{32}, \\
\mu_{1}(\{1,2,3\})=\mu_{1}(\{1\})=\mu_{1}(\{2\})=\mu_{1}(\{3\})=0, \\
\mu_{1}(\{1,2\})=\mu_{1}(\{1,3\})=\frac{9}{32}, \quad \mu_{1}(\{2,3\})=\frac{8}{32} .
\end{gathered}
$$

Thanks to the symmetries of this measure, it is easy to check that the measure is strictly negatively associated.

This example would have been a counterexample to Conjecture 1 had it not been for the caveat that the nonzero elements of the rank sequence must form an interval. However, since the example is strictly negatively associated this can easily be circumvented.

Theorem 1. If $\mu_{1}$ is strictly negatively associated then there exists a $\delta>0$ such that if $\sup _{A \in B_{n}}\left|\mu_{1}(A)-\mu_{2}(A)\right|<\delta$ then $\mu_{2}$ is strictly negatively associated.

Proof. We immediately see that each $C(f, g)$ is changed continuously in $\mu$, in fact, they are polynomials in $\mu\left(X_{i}\right)$ for $X_{i} \in B_{n}$. As noted above, we only need to check that $C(f, g)$ is negative for the set of pairs of indicator functions of upsets, and since this set of pairs is finite, all $C(f, g)$ will remain negative for small enough $\delta$.

By Theorem 1 we can disturb our measure $\mu_{1}$ to obtain a measure with only nonzero elements in the rank sequence, while still being negatively associated. An explicit example of such a measure is given in Example 2, below.

Example 2. We have

$$
\begin{gathered}
\mu_{2}(\varnothing)=\frac{6}{32}, \\
\mu_{2}(\{1,2,3\})=\mu_{2}(\{1\})=\mu_{2}(\{2\})=\mu_{2}(\{3\})=\frac{1}{32}, \\
\mu_{2}(\{1,2\})=\mu_{2}(\{1,3\})=\frac{7}{32}, \quad \mu_{2}(\{2,3\})=\frac{8}{32} .
\end{gathered}
$$

Again it is easy to explicitly verify that this measure is negatively associated. 
The caveat that the rank sequence should form an interval was introduced in [3] to eliminate trivial examples, but since the set of strictly negatively associated measures forms an open set, this does not give the desired consequences.

\section{References}

[1] BorceA, J., BrändÉn, P. ANd Liggett, T. M. (2007). Negative dependence and the geometry of polynomials. Available at http://www.arxiv.org/abs/0707.2340.

[2] Liggett, T. M. (1997). Ultra logconcave sequences and negative dependence. J. Combin. Theory Ser. A 79, 315-325.

[3] Pemantle, R. (2000). Towards a theory of negative dependence. Probabilistic techniques in equilibrium and nonequilibrium statistical physics. J. Math. Phys. 41, 1371-1390. 